\title{
La creatividad en jóvenes con trastorno de conducta disruptiva
}

\author{
José Miguel García Ramírez. Universidad de Granada \\ Recepción: 29 de julio de 2015 | Revisión: 1 de agosto de 2015 | Aceptación/Publicación: 3 de agosto de 2015 \\ Correspondencia: miguelgr@ugr.es \\ Citar: Garcia-Ramirez, JM. (2015). La creatividad en jóvenes con trastornos de conducta disruptiva. ReiDoCrea, 4, 213-218. \\ http://hdl.handle.net/10481/????
}

\begin{abstract}
Resumen: Las personas jóvenes con trastornos de conductas disruptivas presentan perfiles de creatividad bajos que influyen en sus procesos cognitivos de percibir y entender las relaciones interpersonales en la sociedad. Método: Es un estudio de investigación de carácter exploratorio, en el que participaron 10 jóvenes con trastorno de conducta disruptiva, de los cuales 5 participaron activamente en el Programa Integral de Intervención con Jóvenes en los Centros Penitenciarios. Estos constituyeron el grupo experimental; todos los participantes realizaron la Prueba de Imaginación Creativa (Artola, Barraca, Mosteiro, Ancillo, Poveda y Sánchez, 2012) para comprobar si existían diferencias en sus perfiles de imaginación creativa debidas a la participación en el programa. Resultados: El análisis estadístico mostró que existían diferencias significativas entre los resultados obtenidos de los participantes del grupo experimental respectos a los del grupo control. Conclusiones: El programa integral de intervención con jóvenes en Centros Penitenciarios influye positivamente en los perfiles de la imaginación creativa de las personas jóvenes con trastornos de conducta disruptiva.
\end{abstract}

Palabras clave: Conducta disruptiva | Imaginación creativa

\section{CREATIVITY IN YOUTH WITH DISRUPTIVE BEHAVIOR DISORDERS}

Abstract: Youth with disruptive behavior disorders have low profiles of creativity which influences their cognitive processes of perceiving and understanding interpersonal relationships in society. Method: In this study of exploratory research, which involved 10 men with disruptive behavior disorder, 5 of them participated actively in the Comprehensive Intervention Program for Youth in Correctional Facilities. These 5 participants constituted the experimental group. All of the participants completed the test of Creative Imagination (Artola, Barraca, Mosteiro, Ancillo, Poveda, \& Sánchez, 2012) to determine whether there were differences in their creative imagination profiles due to participation in the program. Results: Statistical analysis showed that there were significant differences between the results of participants in the experimental group compared to the control group. Conclusions: Comprehensive Intervention Programs for Youth in Correctional Facilities positively influence the profiles of creative imagination of young people with disruptive behavior disorders.

Keywords: Disruptive Behavior | Creative Imagination

\section{Introducción}

La criminalidad juvenil se ha incrementado en los últimos años; esto se debe, principalmente, a situaciones de exclusión social, abandono escolar o modificaciones estructurales de la familia; aunque esos factores se encuentren frecuentemente entre las causas sociales de la delincuencia no son los únicos. La delincuencia juvenil es considerada un fenómeno de desviación e inadaptación social, producto del fracaso de adaptación a las demandas sociales. (Ortega, 2010)

La mayoría de las investigaciones realizadas sobre la delincuencia juvenil señalan una serie de factores personales que la caracterizan, entre los que se encuentran la impulsividad, el afán de protagonismo, el fracaso escolar, el consumo de drogas, la baja autoestima, la desestructuración familiar, la falta de afectividad, la agresividad, la ausencia de habilidades sociales, el desequilibrio emocional, la inadaptación social y la frustración. El estudio de esos factores de carácter cognitivo-interpersonal han ayudado a establecer programas de prevención y reeducación, pero aún así sigue aumentado la delincuencia juvenil. La adolescencia es una etapa difícil en el desarrollo humano, un elevado porcentaje de adolescentes admiten haber cometido algún tipo de delito alguna vez en su vida. (Boada y Casas, 2010) 
El fracaso escolar es uno de los factores que ha agravado la situación. El sistema educativo no dispone de los medios necesarios para ofrecer una enseñanza adaptada a los intereses, motivaciones y capacidades de toda la población juvenil, lo que influye en el fracaso y abandono escolar sin haber adquirido las competencias necesarias para mantenerse, con garantías de éxito, en la sociedad; las escuelas no sólo tienen que dar una educación de calidad si no que tienen que construir un marco de análisis para entender la situación y los retos más actuales del planteamiento en pro de una educación inclusiva. (Echeita, 2006)

La educación inclusiva e integral permite que todos sus miembros, tanto alumnado como profesorado, se sientan con pleno derecho y valorados tanto en el contexto como en la comunidad; por lo tanto se puede aprender de forma distinta, porque todas las personas tienen derecho a aprender en relación a sus intereses y motivaciones. La comunidad educativa pasa a ser el sustento de los derechos fundamentales propios de una sociedad humanista y democrática, para dar respuesta a las necesidades e inquietudes sociales, y acceso a los bienes económicos, culturales y sociales; siendo todas generadora de las medidas preventivas que evitan o reducen la exclusión social. (García, Parrón, Roca y Biarnès, 2008)

Las personas jóvenes de entornos desfavorecidos se hayan en circunstancias especialmente de riesgo propias de habitar barrios marginales, con códigos lingüísticos, éticos y sociales diferentes, y convivir en un ambiente de economía sumergida. Esto influye en el fracaso escolar incidiendo en la autoestima y motivación, pudiendo convertirse en un fracaso personal y social, porque no tienen la oportunidad de aprender los patrones básicos de comportamiento social; también está presente el riesgo de consumo de drogas, de delincuencia y otras conductas inadaptadas, dando lugar a trastornos de comportamiento disruptivo (Valencia-García y García-Ramírez, 2014). Es importante no confundir los conceptos de conducta antisocial y trastorno de conducta con el concepto de delincuencia, ya que no todas las personas con algún trastorno de conducta realizan actos delictivos, así que no se debe identificar como delincuente a una persona que manifieste trastornos de comportamiento y/o convivencia. El comportamiento antisocial se presenta en personas que se educan en familias con múltiples problemas tanto de índole socioeconómica como de estructura y dinámica funcional. Por eso, es muy importante promover la existencia de una red de apoyo social y emocional que puedan proporcionar ayuda a las familias con dificultades (Vélaz de Medrano, 2008).

Las investigaciones realizadas sobre los fenómenos violentos o antisociales por jóvenes destacan el vandalismo, la violencia física y el abuso sexual (Alcántara, López, Castro y López, 2013), los cuales se agravan como consecuencia de una necesidad de cierre cognitivo inadecuado (Arjona-Martín y Garcia-Ramirez, 2014). Aún así, no existe de un patrón de personalidad criminal sino ciertos factores individuales que tienen importancia significativa en la aparición y mantenimiento de las conductas antisociales y/o delictivas (López y López, 2008). También es necesario tener en cuenta que el comportamiento es aprendido, que se aprende mediante la asociación diferencial y el resultado de las asociaciones del individuo le enseñan un modo especifico de comportamiento que socialmente; por lo tanto el comportamiento delictivo es aprendido a través de procesos de interacción y comunicación (Boada y Casas, 2010).

La sociedad, a través de los Centros Penitenciarios, tiene la obligación que ofrecer a las personas jóvenes infractoras una medida con carácter socializador, facilitándoles los instrumentos y recursos necesarios para asegurar el éxito en el proceso de socialización y conseguir un desarrollo integral e inclusivo que les permita su 
reinserción en la sociedad. En recientes investigaciones realizadas a menores infractores se descubrió que sus perfiles de imaginación creativa eran bajos y esto influía en sus procesos cognitivos de percibir y entender las relaciones interpersonales en la sociedad (Lozano-Santos y Garcia-Ramirez, 2014). La creatividad depende de las características personales, habilidades cognitivas, conocimientos técnicos, circunstancias socioculturales y recursos, siendo necesaria para progresar de manera contextualizada y su ausencia no se debe a la falta de idea originales y novedosas, sino a la falta de interés por parte de los demás miembro del entorno (Fiorini y GarciaRamirez, 2013).

En otras investigaciones se han demostrado que la mayoría de las personas, en su niñez, exhiben su potencial creativo el cual, generalmente, es ignorado tanto por padres y madres como por sus educadores que no lo reconocen o perciben así. Esa falta de aceptación por los demás le hace parecer rebelde, dando la impresión de que se trata de un ser antisocial. Finalmente en vez de aceptarse a sí mismo, procuran remodelarse de acuerdo a modelos más convencionales y oculta o inhibe su talento (Craft, 2014) e incluso sus valores (Lara Guardia y Garcia-Ramirez, 2014). Por lo tanto, en sistema penitenciario es necesario promover la imaginación creativa de las personas para que se acepten las diferencias propias de cada individuo, consolidando la sociabilización y reduciendo los comportamientos disruptivos y sobre todo la exclusión social (Ortega, 2010).

La Institución Penitenciaria de España cuenta con un Programa Integral de Intervención con jóvenes internos en la prisión, en el que se fomenta el contacto del interno con su entorno social y tiene dos objetivos: $1^{\circ}$ Equipar a los jóvenes con aquellas habilidades de pensamiento necesarias para un mejor ajuste personal y social; $2^{\circ}$ Mejorar las habilidades interpersonales, la educación, junto con la preparación para la búsqueda de empleo.

Con este estudio de investigación, de carácter exploratorio, se pretende comprobar si el Programa integral de Intervención con jóvenes internos en un Centro Penitenciario influye en los perfiles de la imaginación creativa de jóvenes con problemas de conducta disruptiva; formulando la siguiente hipótesis: El Programa Integral de Intervención con jóvenes internos en un Centro Penitenciario influye positivamente en el perfil de creatividad general de los jóvenes internos con problemas de conducta disruptiva.

\section{Método}

\section{Participantes}

Diez personas jóvenes de 20 y 21 años de edad, diagnosticados de trastorno de conducta disruptiva, que estuvieron internos en un Centro Penitenciario por delitos similares (trafico de drogas y hurto con intimidación y/o agresión); cinco de ellos participaron en el Programa Integral de Intervención con jóvenes internos en el Centro Penitenciario; estos cinco participantes constituyeron el Grupo Experimental, GE; los otros cinco no participaron en ninguno de los programas de inclusión social de los que se ofertan en los Centros Penitenciarios; estos cinco participantes constituyeron el Grupo Control, GC.

\section{Instrumento y procedimiento}

Se utilizó la Prueba de Imaginación Creativa (Artola, Barraca, Mosteiro, Ancillo, Poveda, \& Sánchez, 2012), PIC-A, que es una prueba diseñada para medir algunos 
aspectos de la creatividad, mediante la imaginación y la fantasía por parte del sujeto. La PIC-A consta de cuatro bloques. Tres de esos bloques miden la creatividad verbal o narrativa. El cuarto bloque mide la creatividad figurativa o gráfica. Concretamente el Bloque 1 permite al participante expresar su curiosidad e imaginación; el Bloque 2 sirve para que el participante pueda liberar su espíritu y pensar de forma poco convencional; el Bloque 3 tiene como fin evaluar un aspecto fantasioso de la imaginación; y con el Bloque 4 le permite al participante mostrar la originalidad de su imaginación.

Las instrucciones en cada uno de los bloques se debe leer con los participantes para aclarar las dudas que se presenten. Cada bloque se realiza en aproximadamente 10 minutos, aunque es un tiempo estimativo y puede variar según necesidades del participante.

La prueba se pasó a todos los participantes durante el mes de abril de 2014, en el Laboratorio de Psicología Social de la Facultad de Ciencias de la Educación de la Universidad de Granada. La PIC únicamente se usó para medir los perfiles de creatividad general y compararla entre ambos grupos. Los resultados obtenidos de todos los participantes fueron analizados por el programa estadístico SPSS 20 para comprobar si existían diferencias significativas entre las medias de ambos grupos.

\section{Resultados}

A continuación se procede a analizar estadísticamente los resultados. Primero se realiza la prueba de normalidad de Shapiro-Wilk (tabla 1), siendo la significancia mayor de 0.05 ( $p>.05$ ) para ambos grupos (GE: $p=.814$; GC: $p=.656$ ), lo que confirma que los datos provienen de una distribución normal. Por lo tanto procede realizar un análisis estadístico de comparación de medias a través de la T para muestra independientes.

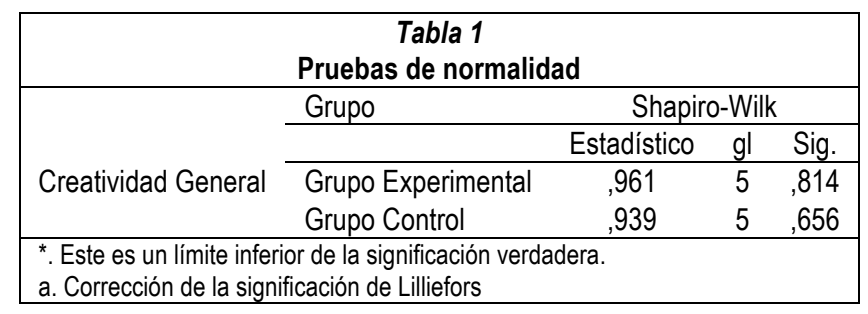

Las medias estadísticas, prueba $\mathrm{T}$, muestra que existen diferencias significativas entre los resultados obtenidos de los participantes del Grupo Control $(M=36.00, S E=6.205)$ y el Grupo Experimental $(\mathrm{M}=62.00$, SE $=2.550, t(8)=3.876, p=.005, r=.80)$; en cuanto a la potencia estadística es alta, dando respuesta al $25 \%$ de la varianza total. También entre los intervalos de confianza para la diferencia no se encuentra el 0 , lo que confirma que el análisis es correcto. Por lo tanto, se puede afirmar que el análisis estadístico de las medias demuestra que la participación en el Programa Integral de Intervención con jóvenes de Sistema Penitenciario con jovenes influye positivamente en los perfiles de creatividad general de los participantes del GE, cumpliéndose la hipótesis. A continuación se muestra con más detalle el análisis estadístico en las tablas 2 y 3.

\begin{tabular}{|l|l|c|c|c|c|}
\hline \multicolumn{7}{|c|}{ Tabla 2 } \\
\hline & Grupo & $\mathrm{N}$ & Media & Desviación típ. & Error típ. de la media \\
\hline Creatividad General & Tratado & 5 & 62,00 & 5,701 & 2,550 \\
\cline { 2 - 7 } & Excluido & 5 & 36,00 & 13,874 & 6,205 \\
\hline
\end{tabular}




\begin{tabular}{|c|c|c|c|c|c|c|c|c|c|c|}
\hline & & & & ba de $n$ & & $\begin{array}{l}\text { la } 3 \\
\text { as indepe }\end{array}$ & dientes & & & \\
\hline & & Prueba & evene & & & & rueba T pare & igualdad de & dias & \\
\hline & & $F$ & Sig. & $t$ & $\mathrm{gl}$ & $\begin{array}{c}\text { Sig. } \\
\text { (bilateral) }\end{array}$ & $\begin{array}{l}\text { Diferencia } \\
\text { de medias }\end{array}$ & $\begin{array}{l}\text { Error típ. de } \\
\text { la diferencia }\end{array}$ & $\begin{array}{r}95 \% \text { Inte } \\
\text { para }\end{array}$ & $\begin{array}{l}\text { confianza } \\
\text { rencia }\end{array}$ \\
\hline & & & & & & & & & Inferior & Superior \\
\hline $\begin{array}{l}\text { Creatividad } \\
\text { General }\end{array}$ & $\begin{array}{l}\text { Se han asumido } \\
\text { varianzas iguales }\end{array}$ & 3,724 & ,090 & 3,876 & 8 & ,005 & 26,000 & 6,708 & 10,531 & 41,469 \\
\hline
\end{tabular}

\section{Discusión y conclusiones}

El análisis estadístico de los datos obtenidos de la Prueba de Imaginación Creativa demuestra que los participantes del Grupo Experimental mostraron mejores perfiles de imaginación creativa general respecto a los miembros del Grupo Control. Por lo tanto, con este estudio exploratorio se reafirman las investigaciones previas de que los programas específicos de intervención implementados por la Institución Penitenciaria influyen positivamente en las personas internas que participan. Aún así estos datos también pueden tener otra interpretación, ya que el análisis puede ser el resultado de la inhibición de la imaginación creativa de los participantes del Grupo Control, porque estos jóvenes internos no recibieron la misma atención que los demás agravándose su situación tanto de precariedad social como de falta de expectativas (Ortega, 2010).

De todas maneras, este estudio exploratorio sólo muestra un análisis de unos pocos casos de jóvenes que han estado internos en un Centro Penitenciario y que previamente habían sido diagnosticado de trastornos de conducta disruptiva. Estos jóvenes después de participar de un programa especifico de inclusión social mostraron unos perfiles de imaginación creativa normales a la mayoría de la población en general. Lo que les permite ver la realidad desde su propio punto de vista, no como una reproducción impuesta, así pueden producir sus propias ideas y expresarse con libertad, sintiéndose parte de la sociedad. También el hecho de tener control sobre la necesidad de cierre cognitivo les ayuda a retomar y superar con éxito una posible recaída.

Para concluir, los programas específicos de inclusión social implementados por la Institución Penitenciaria son necesarios para abordar con eficacia la situación de exclusión social en la que están inmersos las personas jóvenes internas con trastornos de conducta disruptiva. Estos programas ayudan a estos jóvenes a desarrollar: su potencial creativo, su fluidez de expresión, sus ideas o respuestas ante situaciones o problemas determinados. También les ayuda a desarrollar la flexibilidad de su pensamiento, la originalidad de las ideas y la posibilidad de buscar soluciones o respuestas fuera de lo común, pero adecuadas a la situación que se les plantea y controlando adecuadamente la necesidad del cierre cognitivo (Fiorini y GarciaRamirez, 2013; Craft, 2014; Hazir y Garcia-Ramirez, 2015).

\section{Referencias}

Alcántara López, M., López Soler, C., Castro Sáez, M. y López García, J. (2013). Alteraciones psicológicas en menores expuestos a violencia de género: prevalencia y diferencias de género y edad. Anal. de Psicología, 29(3), 741747. http://dx.doi.org/10.6018/analesps.29.3.171481

Arjona Martín, N. y Garcia-Ramirez, J.M. (2014). Los efectos del sexismo no se olvidan ni inmediatamente ni permanentemente. ReiDocrea, 3, 267-272. http://hdl.handle.net/10481/32861

Artola, T., Barraca, J., Mosteiro, P., Ancillo, I., Poveda, B. y Sánchez, N. (2012). Prueba de Imaginación Creativa para Adultos. Madrid: TEA.

Boada, M. y Casas, F. (2010). Educación y jóvenes ex-tutelados: revisión de la literatura científica española. Educación $X X I, \quad 13, \quad 117-138 . \quad$ http://e-spacio.uned.es/fez/eserv.php?pid=bibliuned:EducacionXXI-2010-13-25050\&dsID=Documento.pdf 
Craft, A. (2014). Wise Humanising Creativity: a goal for inclusive education. Revista de Educación Inclusiva, 7(1), 3-15. http://www.revista-educacion-inclusiva.es/numeros/anteriores

Echeita Sarrionandia, G. (2011). Educación para la inclusión o educación sin exclusiones. Madrid: Narcea.

Fiorini, M. y García-Ramírez, J.M. (2013). Cap. 5: Técnicas de grupo y creatividad aplicadas en el ámbito universitario, en Villena, MD., Muñoz, A. Recursos para tutoría en el aula universitaria. Granada: Editorial Universidad de Granada.

García, M.J., Parrón, M.R., Roca, J.S., y Biarnès, A.V. (2008). El proceso de desinternamiento de jóvenes ex-tutelados para favorecer una inserción sociolaboral positiva. Análisis de la situación actual en los centros residenciales de acción educativa de Cataluña. Bordón, 60(3), 49-65. http://recyt.fecyt.es/index.php/BORDON/article/view/29000/15461

Hazir, M., \& García-Ramírez, J.M. (2015). Creativity \& Cognitive Closure. Reidocrea, 4, 18-23. http://hdl.handle.net/10481/34628

Lara Guardia, N.G. y Garcia-Ramirez, JM. (2014). Educación en valores en estudiantes de Educación Secundaria Obligatoria. ReiDoCrea, 3, 285-308. http://digibug.ugr.es/handle/10481/32878

López, JR. y López, C. (2008). Conducta antisocial y delictiva en la adolescencia. Universidad de Murcia: EDITUM.

Lozano Santos, D., y Garcia-Ramirez, J.M. (2014). La imaginación en menores de contextos educativos marginados. ReiDoCrea, 3, 261-266. http://digibug.ugr.es/handle/10481/32855

Ortega Esteban J. (2010). Delincuencia, reformatorio y educación liberadora. Salamanca: AMERU.

Valencia-García, N., y García-Ramírez, J.M. (2014). A través de la lectura. ReiDocrea, 3, 273-284. http://digibug.ugr.es/handle/10481/32877

Vélaz de Medrano Ureta, M.C. (2008). Intervención educativa y orientadora para la inclusión social de menores en riesgo. Factores escolares y socioculturales. Madrid: UNED. 